\title{
Home Energy Management Machine Learning Prediction Algorithms: A Review
}

\author{
Ohoud Almughram ${ }^{1, *}$, Bassam Zafar², Sami Ben Slama \\ 1,2 Information Systems Department, FCIT, King Abdulaziz University, Jeddah, Saudi Arabia \\ ${ }^{3}$ Faculty of Applied Studies, King Abdulaziz University, Jeddah, Saudi Arabia \\ *Email: omalmughram@stu.kau.edu.sa
}

\begin{abstract}
Renewable energies are being introduced in countries around the world to move away from the environmental impacts from fossil fuels. In the residential sector, smart buildings that utilize smart appliances, integrate information and communication technology and utilize a renewable energy source for in-house power generation are becoming popular. Accordingly, there is a need to understand what factors influence the accuracy of managing such smart buildings. Thus, this study reviews the application of machine learning prediction algorithms in Home Energy Management Systems. Various aspects are covered, such as load forecasting, household consumption prediction, rooftop solar energy generation, and price prediction. Also, a proposed Home Energy Management System framework is included based on the most accurate machine learning prediction algorithms of previous studies. This review supports research into the selection of an appropriate model for predicting energy consumption of smart buildings.
\end{abstract}

Keywords: Home Energy Management System, Machine Learning algorithm, Prediction, Forecasting, Optimization

\section{INTRODUCTION}

In the last few years, consumers and governments have been increasing the pressure on manufacturers to reduce transportation $\mathrm{CO}_{2}$ emissions and lower oil consumption costs. Photovoltaic (PV) solar and wind power are the most popular Renewable Energy (RnEs) sources [1]. Large private or public entities use RnEs to generate power given the cost of the conventional power infrastructure. Many individual consumers are energy supporters and play an important role in renewable energy development [2].

Smart buildings (SBs) are designed, constructed, planned, and managed differently than conventional buildings in order to save energy, reduce power consumption, and create more sustainable buildings. Although SBs improve quality of life, they face many challenges. An efficient Energy Management System (EMS) helps to reduce peak period demands, lower unnecessary energy consumption, and decrease associated costs. Future EMS development depends on Artificial Intelligence (AI). Furthermore, incorporating multiple agents in a smart microgrid (MG) and machine learning (ML) help to predict index parameters, such as temperature, humidity, and sunlight. There are several types of ML algorithms, including: Linear and Logistic Regression, Random Forest (RF), Decision Tree (DT),
Neural Networks (NN) and Gradient Boosting. Deep Learning (DL) is most effective for analysing data and enhancing SB energy management [3]. In addition, home automation is a potential technology to achieve power performance efficiency. Such automation enhances the flow of power without interruption, solves power demand problems, and coordinates devices with innovative technologies [4].

An efficient Home Energy Management System (HEMS) is affected by ML algorithm prediction accuracy. Currently, it is a challenging task to arrive at precise and reliable predictions [3]. This paper presents a comprehensive review of ML prediction algorithms that have been applied to predict various HEMS parameters, including: load forecasting, household consumption, rooftop solar panel energy generation and price prediction. This paper aims to discuss the effectiveness of ML prediction algorithms in enhancing the overall accuracy and reliability of HEMSs. The review covers the last three years of 2019, 2020, and 2021. Any MAPE greater than three was excluded to avoid lengthy tables and unnecessary comparisons. This study is novel in providing an interrelated article that describes at a glance the most influential studies that aid research planning, cross-disciplinary collaboration, and future directions. 
Section 2 provides a detailed investigation of ML prediction algorithm application in HEMSs for load forecasting, household consumption, rooftop solar panel energy generation and price prediction. In Section 3, HEMS systems are optimized by proposing a hybrid model of the most accurate ML prediction algorithms. Finally, the conclusion sums up the main findings and proposes future work to optimize HEMSs.

\section{MACHINE LEARNING PREDICTION ALGORITHMS IN HEMS}

Building owners and household residents can utilize ML to regulate and predict energy usage [5]. In this regard, individuals are able to effectively manage possible energy consumption, and thus, reduce electricity or power bills. Furthermore, energy generation and management can be key parameters to support consumer and supplier efforts to enhance energy optimization while reducing electricity-related costs.

This section extensively discusses ML prediction algorithms that have been applied for predicting several aspects in HEMS, including: load forecasting, household consumption, rooftop solar panel energy generation and price prediction. The following subsections verify previous studies to determine the applicability of ML prediction algorithms in the HEMS field.

\subsection{LOAD FORECASTING}

The task of load forecasting is usually used to balance demand and supply of energy in HEMS. It is a time-series forecasting method that uses power load as an object to predict future power demand based on historical load fluctuations. The load forecasting depends on the data provided from smart meters and smart sensors equipped with smart MG [5].

Machine learning prediction algorithms and timeseries methods are being exploited to optimize the reliability and accuracy of load forecasting results [6]. Accurate load forecasting is essential for decision making, planning, operating, and improving the economic profits of the grid. For instance, daily planning and scheduling in a smart MG demands dayahead load forecasting of the smart building. The model accuracy will have a considerable impact on several decisions, such as planning for energy transactions, economic scheduling of generating capacity, system security assessment, and scheduling [7].

Load forecasting can be short-, mid-, or long-term power load forecasting depending on the time interval of the historical data [8]. Short-term load forecasting (STLF) forecasts one hour to a week; mid-term load forecasting (MTLF) forecasts one month up to a year; long-term load forecasting (LTLF) forecasts over one year.
The following three tables (Table 1, Table 2, Table 3) review the use of ML prediction algorithms in STLF, MTLF, and LTLF, respectively. Noticeably, there are more studies for STLF than for MTLF and LTLF, because HEMS depends on STLF to plan and make important decisions for residences in hourly or daily bases. Additionally, the most accurate STLF is the Fusion Forecasting (FA) approach, with the lowest MAPE equalling $0.0284 \%$ in Table 1 . The most accurate MTLF is a hybrid model of CA-CA-WSVR with a MAPE of $1.04 \%$, as shown in Table 2 . The most accurate LTLF is a hybrid model of Cascade Forward Backpropagation Neural Network (CFBNN), with a MAPE of $0.1 \%$, as shown in Table 3.

\subsection{HOUSEHOLD CONSUMPTION PREDICTION}

One of the main foundations of smart energy management is the prediction of energy consumption. The diversity of pattern usage among households and total energy consumption are two features of power use in residential buildings. Better prediction of energy and peak demand are vital for appropriate planning and improvements of distribution systems and power generation, considering that the consumption of energy fluctuates with different appliances. Thus, ensuring stable power demand forecasting is important for maintaining resources [42].

Machine learning techniques have several forecasting applications in household energy consumption using the data coming from smart meter. Different classification, clustering, and regression models analyse smart meter data for more accurate prediction of electrical appliance consumption. Table 4 reviews the ML applications for household consumption prediction in different periods (hourly, daily, weekly, monthly, and annually). The review shows that DB-Net is the most accurate model, with a MAPE of $0.12 \%$ on an hourly basis. The hybrid model of rough set and Deep Neural Network exceeds its counterparts with a MAPE $=0.029 \%$ on a daily basis. For the weekly household consumption prediction, it was noted that there is a lack of models with a MAPE less than three. However, the simple tree has the lowest MAPE at $0.96 \%$. For the monthly periodicity, the EMD-SSM counterparts the other models with a MAPE of $0.0216 \%$. Finally. the Gaussian radial basis function kernel SVR is the most accurate model for annual household consumption prediction.

\subsection{PREDICTION OF ROOFTOP SOLAR PANELS ENERGY GENERATION}

In Smart MG, predicting the energy of PV solar generation is essential to optimize the HEMSs. Actually, PV penetration rates raise continuously due to the nature of solar sources. Accordingly, PV energy prediction is an important component to manage uncertainty and ensuring the system stability [59]. 
Uncertainty and fluctuation issues affect the control of the whole Smart MG. Thus, the accurate prediction of the solar supply is a process that needs an on-site measurements and control [60]. Table 5 reviews the use of ML prediction algorithms in predicting the energy generated from the rooftop solar panels in different time series. It was noted that quasi-Newton algorithm is the most precise algorithm on an hourly basis prediction. For the daily prediction, the hybrid model consisting of k-means and support vector regression outstands the other counterparts with the lowest MAPE, whereas adaptive neuro-fuzzy inference systems (ANFIS) are the best for weekly prediction of PV energy generation. For the monthly prediction, no ML prediction algorithm exists with a MAPE less than three. For annual prediction of the PV energy generation, adaptive time-varying parameters discrete grey mode (ATDGM) is the only algorithm with a MAPE less than three.

Table 1: Different ML Algorithms’ Accuracy for Short Term Load Forecasting

\begin{tabular}{|c|c|c|c|}
\hline \begin{tabular}{|c|} 
Algorithms \\
\end{tabular} & MAPE & $\begin{array}{c}\text { Algorithms } \\
\end{array}$ & MAPE \\
\hline $\begin{array}{l}\text { C-Shape Clustering, LSTM networks, and } \\
\text { Xgboost [5] }\end{array}$ & $1.9 \%$ & Multi-scale convolutions (MS-CNN) [14] & $0.98 \%$ \\
\hline $\begin{array}{l}\text { Bi-directional Long Short-term Memory } \\
\text { (Bi-LSTM) Neural Network, Attention } \\
\text { Mechanism (AM), and Rolling Update } \\
\text { (RU) [9] }\end{array}$ & $1.03 \%$ & (EMDHR-SVR-BPNN) [15] & $0.04 \%$ \\
\hline $\begin{array}{l}\text { Generalized Regression Neural Network } \\
(\text { GRNN) [6] }\end{array}$ & $2.41 \%$ & \multirow[t]{2}{*}{$\begin{array}{l}\text { Multi-temporal-spatial-scale Temporal } \\
\text { Convolutional Network (MTCN) [16] }\end{array}$} & \multirow[t]{2}{*}{$1.89 \%$} \\
\hline $\begin{array}{l}\text { Multivariable Linear Regression (MLR) } \\
{[10]}\end{array}$ & $2.88 \%$ & & \\
\hline $\begin{array}{l}\text { A hybrid ANN-based with modified Error } \\
\text { Distribution Estimated (mEDE) in day- } \\
\text { ahead load-forecasting (DALF) model [7] }\end{array}$ & $1.24 \%$ & Stacking Fusion model [17] & $0.88 \%$ \\
\hline $\begin{array}{l}\text { Bidirectional Recurrent Neural Network } \\
(\text { Bi-RNN) and Deep Belief Network } \\
(\mathrm{DBN})[8]\end{array}$ & $1.95 \%$ & $\begin{array}{l}\text { (STLF-IGEP_ALR) Improved Gene Expression } \\
\text { Programming and Abnormal Load Recognition } \\
{[18]}\end{array}$ & $0.638 \%$ \\
\hline $\begin{array}{l}\text { Adaptive weight allocation strategy AWAS } \\
\text { [11] }\end{array}$ & $1.62 \%$ & Weighted k-nearest neighbor [19] & $0.136 \%$ \\
\hline FA fusion forecasting approach [12] & $0.028 \%$ & Deep Neural Networks (DNN) [20] & $2.08 \%$ \\
\hline Fuzzy time series (FTS) [13] & $1.32 \%$ & Parallel LSTM-CNN Network (PLCNet) [21] & $1.48 \%$ \\
\hline
\end{tabular}

Table 2: Different ML Algorithms’ Accuracy for Mid Term Load Forecasting

\begin{tabular}{|l|l|}
\hline \multicolumn{1}{|c|}{ Algorithms } & MAPE \\
\hline $\begin{array}{l}\text { Double Neural Network Autoregressive } \\
\text { eXogenous (D-NAX) [22] }\end{array}$ & $1.90 \%$ \\
\hline $\begin{array}{l}\text { Multilayer Feed-Forward NN } \\
\text { (MFFNN) and Grasshopper } \\
\text { Optimization Algorithm (GOA) [23] }\end{array}$ & $1.06 \%$ \\
\hline $\begin{array}{l}\text { Kernel principal component analysis } \\
\text { (KPCA) and BPNN [24] }\end{array}$ & $1.39 \%$ \\
\hline $\begin{array}{l}\text { SVR and Symbiotic Organism Search } \\
\text { Optimization (SOSO) [25] }\end{array}$ & $1.39 \%$ \\
\hline $\begin{array}{l}\text { Two-step correlation analysis and } \\
\text { wavelet support vector regression } \\
\text { CA-CA-WSVR [26] }\end{array}$ & $1.04 \%$ \\
\hline $\begin{array}{l}\text { Recurrent artificial neural network } \\
\text { (RANN) [27] }\end{array}$ & $1.71 \%$ \\
\hline
\end{tabular}

Table 3: Different ML Algorithms' Accuracy for Long Term Load Forecasting

\begin{tabular}{|l|l|}
\hline Algorithms & MAPE \\
\hline Fuzzy Bayesian [28] & $2.35 \%$ \\
\hline $\begin{array}{l}\text { Least-square Support Vector Machine } \\
\text { (LSSVM) [29] }\end{array}$ & $0.13 \%$ \\
\hline $\begin{array}{l}\text { Artificial Neuro-Fuzzy Intelligent System } \\
\text { (ANFIS) [30] }\end{array}$ & $0.53 \%$ \\
\hline $\begin{array}{l}\text { Multivariate adaptive regression splines } \\
\text { (MARS) [31] }\end{array}$ & $1.3 \%$ \\
\hline Harris Hawk Optimization (HHO) [32] & $1.5 \%$ \\
\hline $\begin{array}{l}\text { Cascade Forward Backpropagation Neural } \\
\text { Network (CFBNN) [33] }\end{array}$ & $0.1 \%$ \\
\hline The Prophet model [34] & $1.75 \%$ \\
\hline $\begin{array}{l}\text { Auto-Regressive Integrated Moving Average } \\
\text { (ARIMA), ANN, and SVR [35] }\end{array}$ & $0.97 \%$ \\
\hline
\end{tabular}


Table 4: Different ML Algorithms' Accuracy for House Hold Consumption Prediction

\begin{tabular}{|c|c|c|}
\hline & Algorithms & MAPE \\
\hline \multirow{2}{*}{ 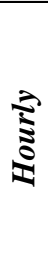 } & $\begin{array}{l}\text { SPEC; a hybrid model integrates } \\
\text { Artificial Neural Networks } \\
\text { (ANN), Ridge Regression, and } \\
\text { Random Forest Regression [36] }\end{array}$ & $1.5 \%$ \\
\hline & $\begin{array}{l}\text { Dilated CNN with bidirectional } \\
\text { long short-term memory (DB- } \\
\text { Net) [37] }\end{array}$ & $0.12 \%$ \\
\hline \multirow{6}{*}{$\overrightarrow{\frac{\pi}{5}}$} & $\begin{array}{l}\text { Fuzzy C-Means and Ensemble } \\
\text { Empirical Mode Decomposition } \\
\text { (FCM, EEMD) [38] }\end{array}$ & $5.21 \%$ \\
\hline & $\begin{array}{l}\text { Household electrical energy } \\
\text { consumption (HousEEC) [39] }\end{array}$ & $0.23 \%$ \\
\hline & $\begin{array}{l}\text { RF, Extra Tree and K-nearest } \\
\text { neighbor regression [40] }\end{array}$ & $0.349 \%$ \\
\hline & DNN [41] & $2.97 \%$ \\
\hline & Rough set and DNN [42] & $0.029 \%$ \\
\hline & ANN [45] & $2.2 \%$ \\
\hline$\frac{\overrightarrow{2}}{2}$ & Simple tree $[46]$ & $0.96 \%$ \\
\hline \multirow{8}{*}{$\frac{\sqrt{2}}{\sqrt[5]{\frac{1}{2}}}$} & Jaya-ELM [47] & $2.41 \%$ \\
\hline & $\begin{array}{l}\text { Synthetic minority technique } \\
\text { ENN and SVM (SMOTE-ENN- } \\
\text { SVM) [48] }\end{array}$ & $0.930 \%$ \\
\hline & $\begin{array}{l}\text { Gradient Boosting Machine } \\
\text { (GBM) [49] }\end{array}$ & $0.1465 \%$ \\
\hline & $\begin{array}{l}\text { Long short-term memory (LSTM) } \\
\text { [50] }\end{array}$ & $0.07 \%$ \\
\hline & $\begin{array}{l}\text { DNN selecting the top } 10 \text { (DNN- } \\
\text { T10) [51] }\end{array}$ & $2.19 \%$ \\
\hline & $\begin{array}{l}\text { EMD and State Space Model } \\
\text { (SSM) [52] }\end{array}$ & $0.0216 \%$ \\
\hline & Seasonal ANN (Model-4) [53] & $1.97 \%$ \\
\hline & $\begin{array}{l}\text { Three ANN techniques (BPNN, } \\
\text { Radial Bases Function Network } \\
\text { RBFNN and Extreme Learning } \\
\text { Machine ELM) [54] }\end{array}$ & $0.99 \%$ \\
\hline \multirow{4}{*}{ 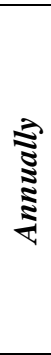 } & Nonlinear regression models [55] & $1.79 \%$ \\
\hline & $\begin{array}{l}\text { K-medoids clustering, SVM and } \\
\text { ANN [56] }\end{array}$ & $0.52 \%$ \\
\hline & $\begin{array}{l}\text { Gaussian radial basis function } \\
\text { kernel SVR [57] }\end{array}$ & $0.44 \%$ \\
\hline & $\begin{array}{l}\text { GM }(1,1) \text { model, DGM }(2,1) \\
\text { model Regression Analysis, and } \\
\text { Polynomial Model Polynomial } \\
\text { Regression [58] }\end{array}$ & $0.47 \%$ \\
\hline
\end{tabular}

Table 5: Different ML Algorithms' Accuracy for Predicting Rooftop Solar Energy Generation

\begin{tabular}{|c|c|c|}
\hline & Algorithms & MAPE \\
\hline \multirow{6}{*}{ 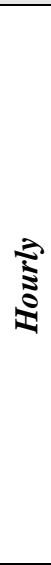 } & $\begin{array}{l}\text { EMD, Sine Cosine Algorithm } \\
\text { (EMD, SCA, ELM) technique } \\
\text { [59] }\end{array}$ & $1.88 \%$ \\
\hline & $\begin{array}{l}\text { Genetic Algorithm-based SVM } \\
\text { (GASVM) [60] }\end{array}$ & $1.70 \%$ \\
\hline & $\begin{array}{l}\text { Davidon-Fletcher-Powell (DFP) } \\
\text { quasi-Newton algorithm } \\
\text { [61] }\end{array}$ & $\begin{array}{l}0.00195 \\
\%\end{array}$ \\
\hline & Neural Network (NN) [62] & $0.55 \%$ \\
\hline & Random Forest (RF) [63] & $\begin{array}{l}0.0054 \\
\%\end{array}$ \\
\hline & $\begin{array}{l}\text { A hybrid improved multi-verse } \\
\text { optimizer algorithm (HIMVO) } \\
\text { [64] }\end{array}$ & $2.71 \%$ \\
\hline \multirow{3}{*}{ ลें } & $\begin{array}{l}\text { Support Vector Regression (SVR) } \\
\text { [65] }\end{array}$ & $2.95 \%$ \\
\hline & k-means and SVR [66] & $1.79 \%$ \\
\hline & $\begin{array}{l}\text { Seasonal ARIMA and Random } \\
\text { Vector Functional Link NN } \\
\text { (SARIMA- RVFL ) [67] }\end{array}$ & $2.723 \%$ \\
\hline$\frac{\sqrt{2}}{\frac{\pi}{2}}$ & $\begin{array}{l}\text { Adaptive Neuro-Fuzzy Inference } \\
\text { Systems (ANFIS) [68] }\end{array}$ & $0.14 \%$ \\
\hline 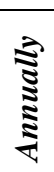 & $\begin{array}{l}\text { Adaptive Time-varying } \\
\text { parameters Discrete Grey Mode } \\
\text { ATDGM [69] }\end{array}$ & $2.98 \%$ \\
\hline
\end{tabular}

\subsection{PRICE PREDICTION}

The environmental and economic advantages of RE have grown significantly over the past decade. Likewise, system efficiency has a significant impact on energy cost savings [70]. Demand response (DR) applications can modify electricity usage patterns. This modification should be profitable for consumers as well as the utility and service providers by applying some technology or other methods. There are diverse electricity tariffs, such as peak pricing (PP), critical peak pricing (CPP), time of use pricing (ToU) and real time pricing (RTP). In scheduling systems, ToU or/and RTP are more common [71]. Thus, Table 6 summarizes the use of ML prediction algorithms in price prediction using RTP and ToU. The algorithms are compared in terms of savings percentage. The comparison shows that the energy storage system management controller (LSEMC) and load scheduling based on heuristic algorithms is the most accurate model in terms of RTP. The rainfall algorithm is superior in terms of ToU tariff. 
Table 6: Different ML Algorithms’ Accuracy for Price Prediction

\begin{tabular}{|c|l|l|}
\hline $\begin{array}{c}\text { Pricing } \\
\text { Strategies }\end{array}$ & \multicolumn{1}{|c|}{ Algorithms } & \multicolumn{1}{c|}{$\begin{array}{c}\text { Savings } \\
\%\end{array}$} \\
\hline \multirow{5}{*}{} & $\begin{array}{l}\text { Load Scheduling and Energy } \\
\text { Management Controller } \\
\text { (LSEMC) based on heuristic } \\
\text { algorithms [70] }\end{array}$ & $58.69 \%$ \\
\cline { 2 - 3 } & DR algorithm [71] & $38 \%$ \\
\cline { 2 - 3 } & $\begin{array}{l}\text { Mixed-Integer Linear } \\
\text { Programming (MILP) [72] }\end{array}$ & $10.55 \%$ \\
\cline { 2 - 3 } & $\begin{array}{l}\text { Modified particle swarm } \\
\text { optimisation (MPSO) } \\
\text { algorithms [73] }\end{array}$ & $20.43 \%$ \\
\hline \multirow{2}{*}{$\approx$} & Rainfall algorithm [74] & $31.335 \%$ \\
\cline { 2 - 3 } & $\begin{array}{l}\text { Deep reinforcement learning } \\
\text { (DRL) [75] }\end{array}$ & $11 \%$ \\
\hline
\end{tabular}

\section{OPTIMIZING THE MANAGEMENT OF HEMS}

A proposed hybrid model incorporating the most accurate ML algorithms to optimize the HEMS is designed in Figure 1. The historical data, including load from smart meter, generated energy from the PV solar panels, and appliance consumption patterns data will be sent to the HEMS.

The data will be analysed using the appropriate ML prediction algorithm that provides more accurate results for that data type. Then, critical decisions will be made based on the predicted information, such as rescheduling certain appliances or activities, DR, and price negotiation, as summarized in Table 7.

Table 7: Algorithms and Their Possible Decisions and Suggestions

\begin{tabular}{|c|c|c|c|}
\hline $\begin{array}{c}\text { Algorith } \\
\text { m }\end{array}$ & Prediction & $\begin{array}{c}\text { Periodici } \\
\text { ty }\end{array}$ & $\begin{array}{c}\text { Decision/Sugges } \\
\text { tion }\end{array}$ \\
\hline $\begin{array}{c}\text { Fusion } \\
\text { Forecasti } \\
\text { ng }\end{array}$ & $\begin{array}{c}\text { Load } \\
\text { forecastin } \\
\text { g }\end{array}$ & STLF & $\begin{array}{c}\text { Reschedule for } \\
\text { peak loads and } \\
\text { DR }\end{array}$ \\
\hline $\begin{array}{c}\text { K-means } \\
\text { and SVR }\end{array}$ & $\begin{array}{c}\text { Rooftop } \\
\text { solar } \\
\text { generation }\end{array}$ & Daily & $\begin{array}{c}\text { DR in case of } \\
\text { PV generation } \\
\text { shortage }\end{array}$ \\
\hline $\begin{array}{c}\text { Rough } \\
\text { set and } \\
\text { DNN }\end{array}$ & $\begin{array}{c}\text { Appliance } \\
\text { consumpti } \\
\text { on }\end{array}$ & Daily & $\begin{array}{c}\text { Decrease } \\
\text { unnecessary } \\
\text { consumption }\end{array}$ \\
\hline $\begin{array}{c}\text { Rainfall } \\
\text { algorith } \\
\text { m }\end{array}$ & $\begin{array}{c}\text { Price } \\
\text { prediction }\end{array}$ & ToU & $\begin{array}{c}\text { Pay during off } \\
\text { peak }\end{array}$ \\
\hline
\end{tabular}

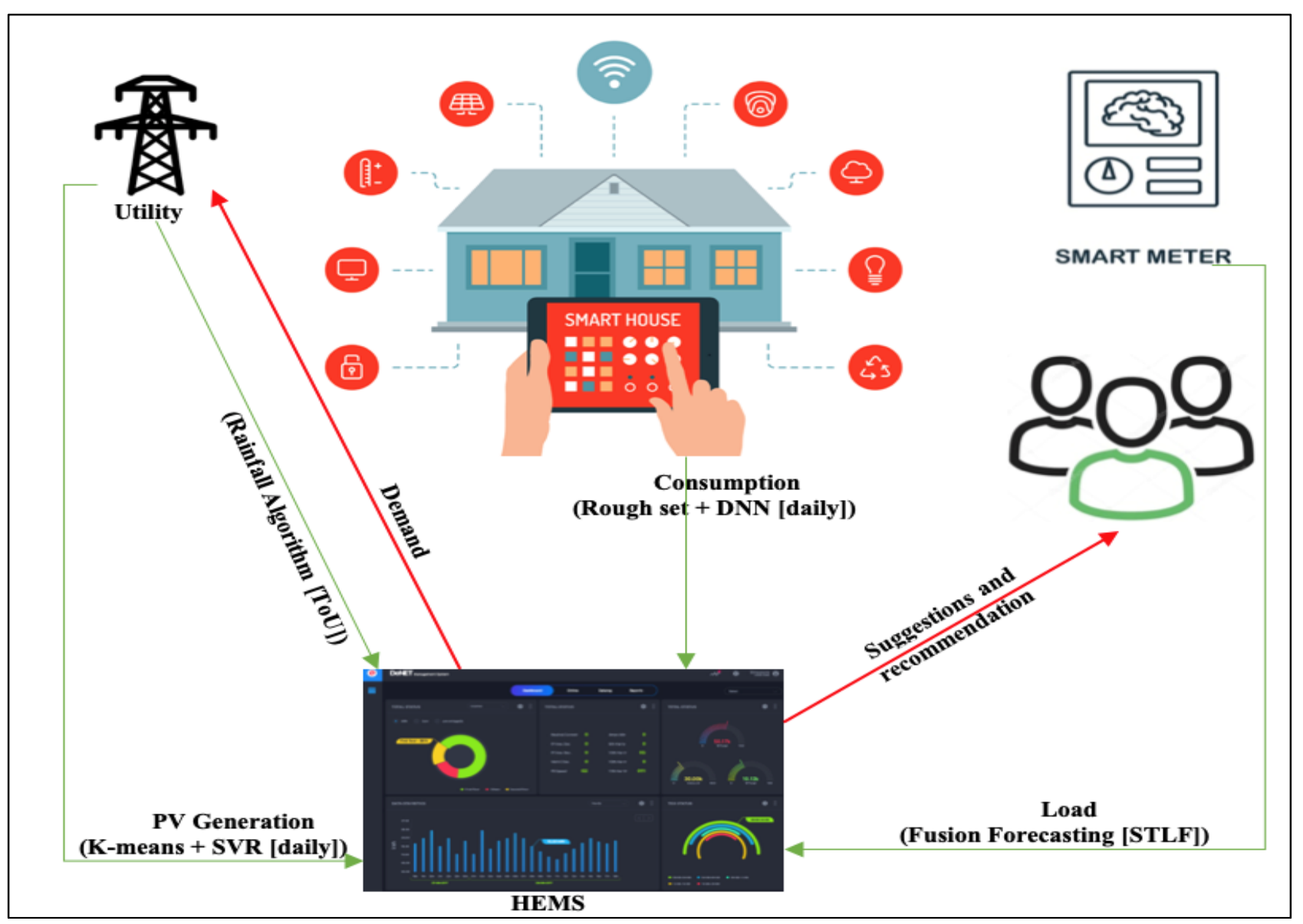

Figure 1: A Schematic of Optimized HEMS Prediction Algorithms Concept 


\section{CONCLUSION}

Accurate prediction in HEMS is essential to managing the DR in residential smart homes and to increase their comfort with affordable electricity bills. This paper reviewed the application of ML prediction algorithms in many aspects in the field of HEMSs. The review shows that reliable load forecasting can effectively balance demand and supply of energy in HEMS. In addition, accurate prediction of household consumption significantly impacts the scheduling for peak demand to ensuring stable power demand which is important for resources maintainability. Furthermore, precise PV energy prediction affects the control of the whole Smart MG by managing the uncertainty and fluctuation issues. While, price prediction can provide energy cost savings.

Also, an optimized HEMS model based on the most accurate ML prediction algorithms was proposed. The model will help manage the energy demand and provide suggestions to the users depending on the predicted information to enhance the overall residents' experience economically and to improve their lifestyle. In the future, the model will be prototyped using multi-layer techniques and a multi-agent system simulation. The accuracy will be compared with similar models to evaluate its functionality and cost effectiveness.

\section{AUTHORS' CONTRIBUTIONS}

O.A planned the presented idea and wrote the manuscript with support from B.Z and S.B who contributed to the original draft preparation, supervised the findings and final revisions.

\section{AKNOWLEGEMENTS}

I would like to show my appreciation to Prof. Bassam and Dr. Sami for their expertise and assistance in writing the manuscript.

\section{REFERENCES}

[1] W. Zhou, C. Lou, Z. Li, L. Lu, and H. Yang, "Current status of research on optimum sizing of standalone hybrid solar-wind power generation systems," Appl. Energy, vol. 87, no. 2, pp. 380-389, 2010.

[2] Z. Maheshwari and R. Ramakumar, "Smart integrated renewable energy systems (SIRES): A novel approach for sustainable development," Energies, vol. 10, no. 8, p. 1145, 2017.

[3] D. Zhang, X. Han, and C. Deng, "Review on the research and practice of deep learning and reinforcement learning in smart grids," CSEE J. Power Energy Syst., vol. 4, no. 3, pp. 362-370, 2018. [4] SHAREEF, Hussain; AL-HASSAN, Eslam; SIRJANI, Reza. Wireless home energy management system with smart rule-based controller. Applied Sciences, 2020, 10.13: 4533.

[5] FAHIMAN, Fateme; ERFANI, Sarah M.; LECKIE,
Christopher. Robust and accurate short-term load forecasting: A cluster oriented ensemble learning approach. In: 2019 International Joint Conference on Neural Networks (IJCNN). IEEE, 2019. p. 1-8.

[6] WU, Zhuochun, et al. A hybrid model based on modified multi-objective cuckoo search algorithm for short-term load forecasting. Applied energy, 2019, 237: 896-909.

[7] AHMAD, Ashfaq, et al. Short-term load forecasting in smart grids: An intelligent modular approach. Energies, 2019, 12.1: 164.

[8] TANG, Xianlun, et al. Application of bidirectional recurrent neural network combined with deep belief network in short-term load forecasting. IEEE Access, 2019, 7: 160660-160670.

[9] WANG, Shouxiang, et al. Bi-directional long shortterm memory method based on attention mechanism and rolling update for short-term load forecasting. International Journal of Electrical Power \& Energy Systems, 2019, 109: 470-479.

[10] LI, Jian, et al. A novel hybrid short-term load forecasting method of smart grid using mlr and lstm neural network. IEEE Transactions on Industrial Informatics, 2020, 17.4: 2443-2452.

[11] ZENG, Pan; JIN, Min; ELAHE, Md Fazla. Shortterm power load forecasting based on cross multimodel and second decision mechanism. IEEE Access, 2020, 8: 184061-184072.

[12] GUO, Weilin, et al. Machine-Learning based methods in short-term load forecasting. The Electricity Journal, 2021, 34.1: 106884.

[13] LI, Chen. A fuzzy theory-based machine learning method for workdays and weekends short-term load forecasting. Energy and Buildings, 2021, 245: 111072. [14] DENG, Zhuofu, et al. Multi-scale convolutional neural network with time-cognition for multi-step short-term load forecasting. IEEE Access, 2019, 7: 88058-88071.

[15] FAN, Guo-Feng, et al. A generalized regression model based on hybrid empirical mode decomposition and support vector regression with back-propagation neural network for mid-short-term load forecasting. Journal of Forecasting, 2020, 39.5: 737756.

[16] YIN, Linfei; XIE, Jiaxing. Multi-temporal-spatialscale temporal convolution network for short-term load forecasting of power systems. Applied Energy, 2021, 283: 116328.

[17] TAN, Zhenqi, et al. Short-term load forecasting based on integration of SVR and stacking. IEEE Access, 2020, 8: 227719-227728.

[18] DENG, Song, et al. Short-term Load Forecasting by Using Improved GEP and Abnormal Load Recognition. ACM Transactions on Internet Technology (TOIT), 2021, 21.4: 1-28.

[19] FAN, Guo-Feng, et al. Application of the weighted k-nearest neighbor algorithm for short-term load forecasting. Energies, 2019, 12.5: 916.

[20] ZHU, Juncheng, et al. Short-term load forecasting for electric vehicle charging stations based on deep learning approaches. Applied sciences, 2019, 9.9: 1723. 
[21] FARSI, Behnam, et al. On short-term load forecasting using machine learning techniques and a novel parallel deep LSTM-CNN approach. IEEE Access, 2021, 9: 31191-31212.

[22] MANZONI, Pietro. Mid-term probabilistic load forecasting with recurrent neural networks. 2021.

[23] Load forecasting based on grasshopper optimization and a multilayer feed-forward neural network using regressive approach

[24] LIU, Zhao, et al. Midterm power load forecasting model based on kernel principal component analysis and back propagation neural network with particle swarm optimization. Big data, 2019, 7.2: 130-138.

[25] ZARE-NOGHABI, Arghavan; SHABANZADEH, Morteza; SANGRODY, Hossein. Medium-term load forecasting using support vector regression, feature selection, and symbiotic organism search optimization. In: 2019 IEEE Power \& Energy Society General Meeting (PESGM). IEEE, 2019. p. 1 -

[26] ALIREZAEI, Hamid Reza; SALAMI, Abolfazl; MOHAMMADINODOUSHAN, Mohammad. A study of hybrid data selection method for a wavelet SVR mid-term load forecasting model. Neural Computing and Applications, 2019, 31.7: 2131-2141.

[27] BAEK, Seung-Mook. Mid-term load pattern forecasting with recurrent artificial neural network. Ieee Access, 2019, 7: 172830-172838.

[28] TANG, Lei, et al. Long-term electricity consumption forecasting based on expert prediction and fuzzy Bayesian theory. Energy, 2019, 167: 11441154

[29] YASIN, Zuhaila Mat; SALIM, Nur Ashida; AB AZIZ, Nur Fadilah. Long term load forecasting using grey wolf optimizer-artificial neural network. In: 2019 7th International Conference on Mechatronics Engineering (ICOM). IEEE, 2019. p. 1-6.

[30] AL-HAMAD, Mohamed Y.; QAMBER, Isa S. GCC electrical long-term peak load forecasting modeling using ANFIS and MLR methods. Arab Journal of Basic and Applied Sciences, 2019, 26.1: 269-282.

[31] KANG, Dahua GANYi WANGShuo YANGChongqing. Long-term load forecasting models based on MARS ANN and LR methods. 2018.

[32] SEKER, Mustafa. Long term electricity load forecasting based on regional load model using optimization techniques: A case study. Energy Sources, Part A: Recovery, Utilization, and Environmental Effects, 2021, 1-23

[33] ARIBOWO, Widi; MUSLIM, Supari. Long-term electricity load forecasting based on cascade forward backpropagation neural network. Journal of Telecommunication, Electronic and Computer Engineering (JTEC), 2020, 12.2: 39-44.

[34] ALMAZROUEE, Abdulla I., et al. Long-term forecasting of electrical loads in kuwait using prophet and holt-winters models. Applied Sciences, 2020, 10.16: 5627 .

[35] KAZEMZADEH, Mohammad-Rasool; AMJADIAN, Ali; AMRAEE, Turaj. A hybrid data mining driven algorithm for long term electric peak load and energy demand forecasting. Energy, 2020,
204: 117948.

[36] CERQUITELLI, Tania; MALNATI, Giovanni; APILETTI, Daniele. Exploiting scalable machinelearning distributed frameworks to forecast power consumption of buildings. Energies, 2019, 12.15: 2933.

[37] KHAN, Noman, et al. DB-Net: A novel dilated CNN based multi-step forecasting model for power consumption in integrated local energy systems. International Journal of Electrical Power \& Energy Systems, 2021, 107023.

[38] CHEN, Yuanyuan; DUAN, Peiyong; LI, Junqing. Hourly electric load forecasting for buildings using hybrid intelligent modelling. In: IOP Conference Series: Earth and Environmental Science. IOP Publishing, 2021. p. 012022

[39] KIPRIJANOVSKA, Ivana, et al. Houseec: Dayahead household electrical energy consumption forecasting using deep learning. Energies, 2020, 13.10: 2672.

[40] MOLDOVAN, Dorin; SLOWIK, Adam. Energy consumption prediction of appliances using machine learning and multi-objective binary grey wolf optimization for feature selection. Applied Soft Computing, 2021, 107745.

[41] AMASYALI, Kadir; EL-GOHARY, Nora. Machine learning for occupant-behavior- sensitive cooling energy consumption prediction in office buildings. Renewable and Sustainable Energy Reviews, 2021, 142: 110714.

[42] LEI, Lei, et al. A building energy consumption prediction model based on rough set theory and deep learning algorithms. Energy and Buildings, 2021, 240: 110886.

[43] VASENIN, D. N., et al. Methods of day ahead load forecasting on the example of a residential area. In: Journal of Physics: Conference Series. IOP Publishing, 2021. p. 012007.

[44] ABERA, Fikirte Zemene; KHEDKAR, Vijayshri. Machine learning approach electric appliance consumption and peak demand forecasting of residential customers using smart meter data. Wireless Personal Communications, 2020, 111.1: 65-82.

[45] GAO, Feng; CHI, Hong; SHAO, Xueyan. Forecasting residential electricity consumption using a hybrid machine learning model with online search data. Applied Energy, 2021, 300: 117393.

[46] CAI, Huiling, et al. Predicting the energy consumption of residential buildings for regional electricity supply-side and demand-side management. IEEE Access, 2019, 7: 30386-30397. [47] ALANEZI, Abdulrahman; P HALLINAN, Kevin; ELHASHMI, Rodwan. Using Smart-WiFi Thermostat Data to Improve Prediction of Residential Energy Consumption and Estimation of Savings. Energies, 2021, 14.1: 187

[48] SON, Hyojoo; KIM, Changwan. A deep learning approach to forecasting monthly demand for residential-sector electricity. Sustainability, 2020, 12.8: 3103

[49] JUNG, Seung-Min, et al. Monthly electric load forecasting using transfer learning for smart cities. Sustainability, 2020, 12.16: 6364. 
[50] HU, Zhineng, et al. Monthly electricity demand forecasting using empirical mode decomposition-based state space model. Energy \& Environment, 2019, 30.7: 1236-1254.

[51] HAMZAÇEBI, Coşkun; ES, Hüseyin Avni; ÇAKMAK, Recep. Forecasting of Turkey's monthly electricity demand by seasonal artificial neural network. Neural Computing and Applications, 2019, 31.7: 2217-2231.

[52] ATUAHENE, Samuel, et al. Artificial Neural Network Based Artificial Intelligent Algorithms for Accurate Monthly Load Forecasting of Power Consumption. London Journal of Research in Science: Natural and Formal, 2019.

[53] WEI, Nan, et al. Conventional models and artificial intelligence-based models for energy consumption forecasting: A review. Journal of Petroleum Science and Engineering, 2019, 181: 106187

[54] HAQ, Ejaz Ul, et al. Forecasting household electric appliances consumption and peak demand based on hybrid machine learning approach. Energy Reports, 2020, 6: 1099-1105.

[55] LI, Xinyi; YAO, Runming. A machine-learningbased approach to predict residential annual space heating and cooling loads considering occupant behaviour. Energy, 2020, 212: 118676.

[56] QIAO, Qingyao; YUNUSA-KALTUNGO, Akilu; EDWARDS, Rodger E. Towards developing a systematic knowledge trend for building energy consumption prediction. Journal of Building Engineering, 2020, 101967.

[57] MASSUCCO, Stefano, et al. A hybrid technique for day-ahead PV generation forecasting using clearsky models or ensemble of artificial neural networks according to a decision tree approach. Energies, 2019, 12.7: 1298

[58] HUSEIN, Munir; CHUNG, Il-Yop. Day-ahead solar irradiance forecasting for microgrids using a long short-term memory recurrent neural network: A deep learning approach. Energies, 2019, 12.10: 1856.

[59] Behera, M. K., Majumder, I., and Nayak, N. (2018). Solar photovoltaic power forecasting using optimized modified extreme learning machine technique. Eng. Sci. Technol. Inter. J. 21, 428-438. doi: 10.1016/j.jestch.2018.04.013

[60] VANDEVENTER, William, et al. Short-term PV power forecasting using hybrid GASVM technique. Renewable energy, 2019, 140: 367-379.

[61] YAO, Xianshuang; WANG, Zhanshan; ZHANG, Huaguang. A novel photovoltaic power forecasting model based on echo state network. Neurocomputing, 2019, 325: 182-189.

[62] ZANG, Haixiang, et al. Day-ahead photovoltaic power forecasting approach based on deep convolutional neural networks and meta learning. International Journal of Electrical Power \& Energy Systems, 2020, 118: 105790.

[63] AL-ROUSAN, Nadia; AL-NAJJAR, Hazem; ALOMARI, Osama. Assessment of predicting hourly global solar radiation in Jordan based on Rules, Trees, Meta, Lazy and Function prediction methods. Sustainable Energy Technologies and Assessments, 2021, 44: 100923.

[64] LI, Ling-Ling, et al. Renewable energy prediction: A novel short-term prediction model of photovoltaic output power. Journal of Cleaner Production, 2019, 228: 359-375

[65] KIM, Taeyoung; KIM, Jinho. A Regional DayAhead Rooftop Photovoltaic Generation Forecasting Model Considering Unauthorized Photovoltaic Installation. Energies, 2021, 14.14: 4256.

[66] AYODELE, T. R., et al. Prediction of global solar irradiation using hybridized k-means and support vector regression algorithms. Renewable Energy Focus, 2019, 29: 78-93.

[67] KUSHWAHA, Vishal; PINDORIYA, Naran M. A SARIMA-RVFL hybrid model assisted by wavelet decomposition for very short-term solar PV power generation forecast. Renewable Energy, 2019, 140: 124-139.

[68] DAWAN, Promphak, et al. Comparison of power output forecasting on the photovoltaic system using adaptive neuro-fuzzy inference systems and particle swarm optimization-artificial neural network model. Energies, 2020, 13.2: 351.

[69] DING, Song; LI, Ruojin; TAO, Zui. A novel adaptive discrete grey model with time-varying parameters for long-term photovoltaic power generation forecasting. Energy Conversion and Management, 2021, 227: 113644.

[70] REHMAN, Ateeq Ur, et al. An Optimal Power Usage Scheduling in Smart Grid integrated with Renewable Energy Sources for Energy Management. IEEE Access, 2021.

[71] AMER, Aya, et al. Home Energy Management System Embedded with a Multi-Objective Demand Response Optimization Model to Benefit Customers and Operators. Energies, 2021, 14.2: 257.

[72] FERNANDEZ, Edstan, et al. A Bi-level optimization-based community energy management system for optimal energy sharing and trading among peers. Journal of Cleaner Production, 2021, 279: 123254.

[73] HOSSAIN, Md Alamgir, et al. Energy management of community energy storage in gridconnected microgrid under uncertain real-time prices. Sustainable Cities and Society, 2021, 66:102658.

[74] ALHASNAWI, Bilal Naji, et al. A Novel RealTime Electricity Scheduling for Home Energy Management System Using the Internet of Energy. Energies, 2021, 14.11: 3191.

[75] LEE, Sangyoon; CHOI, Dae-Hyun. Energy management of smart home with home appliances, energy storage system and electric vehicle: A hierarchical deep reinforcement learning approach. Sensors, 2020, 20.7: 2157. 